\title{
Pacific
}

Journal of

Mathematics

\section{ENTROPY OF A SKEW PRODUCT WITH A $Z^{2}$-ACTION}

\section{KYEWON KOH PARK}




\title{
ENTROPY OF A SKEW PRODUCT WITH A $Z^{2}$-ACTION
}

\author{
KYEWON KOH PARK
}

\begin{abstract}
We consider the entropy of a dynamical system of a skew product $\widehat{T}$ on $X_{1} x X_{2}$ where there is a $Z^{2}$-action on the fiber $X_{2}$. If the $Z^{2}$-action comes from a Cellular Automaton map, then the contribution of the fiber to the entropy of the skew product is the directional entropy in the direction of the integral of a skewing function $\varphi$ from $X_{1}$ to $Z^{2}$.
\end{abstract}

\section{Introduction.}

J. Milnor has defined the notion of directional entropy in the study of dynamics of Cellular Automata [Mi1], [Mi2]. When the notion is applied to a $Z^{n}$ action it is considered to be a generalization of the entropy of non co-compact subgroups of $Z^{n}$.

In the case of a $Z^{2}$-action, we denote the generators of the groups by $\{U, V\}$. Let $P$ be a generating partition under the $Z^{2}$-action. We write $P_{i, j}=U^{i} V^{j} P$. If a subgroup is generated by $U^{p} V^{q}$, then there is a natural way to compute the entropy of $U^{p} V^{q}$ as a $Z$-action on the space. Milnor extended this idea to define the entropy of a vector by embedding $Z^{2}$ to the ambient vector space $R^{2}$ as follows.

$$
h(\vec{v})=\sup _{B: \text { bounded set }} \varlimsup_{t \rightarrow \infty} \frac{1}{t} H\left(\bigvee_{(i, j) \in B+[o, t) \vec{v}} P_{i, j}\right) .
$$

Given a vector $\vec{v}$, we let $\theta_{o}$ be the angle between two vectors $\vec{v}$ and $(1,0)$. Let $w=\frac{1}{\tan \theta_{o}}$ so that $(w, 1)$ is a scalar multiple of the vector $\vec{v}$. It is easy to see that

$$
h(\vec{v})=\lim _{m \rightarrow \infty} \lim _{t \rightarrow \infty} \frac{1}{t} H\left(\bigvee_{j=0-m+j w<i<m+j w}^{[t y]} \bigvee_{i, j}\right),
$$

where $[a]$ denote the greatest integer $\leq a$.

We note that if $\vec{v}=(p, q)$, then $h(\vec{v})=h\left(U^{p} V^{q}\right)$. And it is easy to see that directional entropy is a homogeneous function, that is $h(c \vec{v})=\operatorname{ch}(\vec{v})$ for any $c \in R$.

Directional entropy in the case of a $Z^{2}$-action generated by a Cellular Automaton map has been investigated in $[\mathbf{P a 1}, \mathbf{P a 3}]$ and $[\mathbf{S i}]$. D. Lind 
defined a cone entropy, denoted by $h^{c}(\vec{v})$, of a vector $\vec{v}$. Given a vector $\vec{v}=(x, y)$ and a small angle $\theta$, we consider the vectors $\vec{v}_{\theta}=\left(x_{\theta}, y\right)$ and $\vec{v}_{-\theta}=$ $\left(x_{-\theta}, y\right)$ where $x_{\theta}$ and $x_{-\theta}$ satisfy $\frac{y}{x_{\theta}}=\tan \left(\theta_{o}+\theta\right)$ and $\frac{y}{x_{-\theta}}=\tan \left(\theta_{o}-\theta\right)$ respectively. Cone entropy is defined as follows.

$$
h^{c}(\vec{v})=\lim _{\theta \rightarrow 0} \lim _{n \rightarrow \infty} \frac{1}{n} H\left(\bigvee_{j=0}^{[n y]} \bigvee_{j x_{-\theta} \leq i \leq j x_{\theta}} P_{i, j}\right) .
$$

From the definition, it is clear that we have $h^{c}(\vec{v}) \geq h(\vec{v})$.

We say that a $Z^{2}$-action is generated by a Cellular Automaton if one of the generators of the $Z^{2}$-action, say $V$, is a block map (a finite code) of $U$. That is, $(V(x))_{i}$ depends only on the coordinates $x_{-r}, x_{-r+1}, \ldots, x_{r}[\mathbf{H e}]$. We call $r$ the size of the block map $V$. We will show that in the case of a $Z^{2}$-action generated by a Cellular Automaton map, the directional entropy and the cone entropy are the same (Theorem 1$)$.

Let $\left(X_{1}, \zeta_{1}, \mu_{1}, G\right)$ and $\left(X_{2}, \zeta_{2}, \mu_{2}, H\right)$ be two ergodic measure preserving dynamical systems with finite entropy, where $G$ and $H$ denote the respective group. Given an integrable skewing function $\varphi: X_{1} \rightarrow H$, we define a skew product $G$-action $\widehat{T}$ on $\left(X_{1} \times X_{2}, \zeta_{1} \times \zeta_{2}, \mu_{1} \times \mu_{2}\right)$ such that $\widehat{T}^{g}(x, y)=$ $\left(T^{g} x, F^{\varphi(x)} y\right)$ where $T$ denotes the $G$-action of $X_{1}$ and $F$ denotes the $H$ action on $X_{2}$. When we have $G=H=Z$, then the entropy of $\widehat{T}$ has been extensively studied by many people (e.g. $[\mathbf{A b}],[\mathbf{A d}],[\mathbf{M a}, \mathbf{N e}])$. It is well known in this case that $h(\widehat{T})=h(T)+\left|\int \varphi d \mu\right| h(F)$. The above formula says that, as we expect, the fiber contribution to the entropy is $\left|\int \varphi d \mu\right| h(F)$.

We investigate the entropy of $\widehat{T}$ when $G=Z$ and $H=Z^{2}$. Note that the above formula cannot hold when the acting group on the fiber is a more general group, say $Z^{2}$. First of all, $\int \varphi d \mu$ is in general a vector. Secondly, if the skewing function takes a constant value, say $(1,1)$, then the fiber contribution should come from the entropy of $U V$, not necessarily from the whole $Z^{2}$-action. We prove that if the fiber $Z^{2}$-action is generated by a Cellular Automaton map, then we have the analogous theorem (Theorem 2) to the case when $H=Z$.

We may mention that directional entropy can be also defined in a topological setting. D. Lind constructed an example whose topological entropy does not satisfy the analogue of our Theorem $3[\mathbf{L i}]$. His example involves a $Z^{2}$-action which is not generated by a Cellular Automaton map. It is not clear that Theorem 3 holds for topological entropy when we have a $Z^{2}$-action on the fiber generated by a Cellular Automaton map. Lind's example is not interesting in the measure theoretic sense because it has the trivial invariant measure.

We have constructed a counterexample which does not satisfy Theorem 3 
[Pa2]. For the example we explicitly construct the base transformation and use the $Z^{2}$-action due to Thouvenot [Th] on the fiber. Both of them are constructed by cutting and staking method. It would be interesting to find out how generally Theorem 3 holds. For example, it is unknown if Theorem 3 is true when we have a topological Markov shift which does not satisfy the condition of Corollary 4. We are more interested in the case when the topological Markov shift has 0-entropy as a $Z^{2}$-action.

Although Theorem 2 and 4 are more general than Theorem 1 and 3, we will prove Theorem 1 and 3 because their proofs are easier and more geometric. It is also easy to see the proofs of Theorem 2 and 4 from those of Theorem 1 and 3 .

We would like to thank Professor D. Ornstein for helpful discussions and the Referee for many valuable comments.

\section{Cone entropy.}

Throughout the section we assume that our $Z^{2}$-action is generated by a Cellular Automaton map. We denote by $H^{m}(\vec{v})$

$$
\lim _{n \rightarrow \infty} \frac{1}{n} H\left(\bigvee_{j=0}^{n-1} \bigvee_{i=-m+j w}^{m+j w} P_{i, j}\right)
$$

Note that $H^{m}(\vec{v})$ is independent of the size of the vector $\vec{v}$. Let $\tau$ denote $H\left(P_{0,0}\right)$.

Lemma 1. $H^{m}(\vec{v})=H^{m^{\prime}}(\vec{v})$ if $m, m^{\prime}>2 r+w$.

Proof. Case 1. $\vec{v}$ is not a scalar multiple of $(1,0)$.

Suppose $m^{\prime} \geq m$. Clearly from the definition we have $H^{m^{\prime}}(\vec{v}) \geq H^{m}(\vec{v})$. Hence it is enough to show $H^{m^{\prime}}(\vec{v}) \leq H^{m}(\vec{v})$. Note that

$$
\begin{aligned}
H^{m}(\vec{v}) & =\lim _{n \rightarrow \infty} \frac{1}{n} H\left(\bigvee_{j=0}^{n-1} \bigvee_{-m+j w \leq i \leq m+j w} P_{i, j}\right) \\
& =\lim _{n \rightarrow \infty} \frac{1}{n} \sum_{j=0}^{n-1} H\left(\bigvee_{-m+j w \leq i \leq m+j w} P_{i, j} \mid \bigvee_{0 \leq k<j} \bigvee_{-m+k w \leq i \leq m+k w} P_{i, k}\right)
\end{aligned}
$$




$$
\begin{aligned}
& =\lim _{n \rightarrow \infty} \frac{1}{n}\left\{H\left(\bigvee_{-m \leq i \leq m} P_{i, 0}\right)\right. \\
& +\sum_{j=1}^{n-1} H\left(\bigvee_{j w \leq i \leq(j-1) w+r} P_{i, j} \mid \bigvee_{0 \leq k<j} \bigvee_{k w \leq \imath \leq 2 m+k w} P_{i, k}\right) \\
& +\sum_{j=1}^{n-1} H\left(\bigvee_{(j-1) w-r \leq i \leq j w} P_{\imath, j} \mid \bigvee_{1 \leq k<j-2 m+k w \leq i \leq k w} P_{i, k}\right. \\
& \left.\left.\bigvee \bigvee_{-2 m+j w \leq i \leq-2 m+(j-1) w+r} P_{i, j}\right)\right\} \text {. }
\end{aligned}
$$

We make the following observations:

(1)

$$
\lim _{n \rightarrow \infty} \frac{1}{n} H\left(\bigvee_{-m \leq i \leq m} P_{i, 0}\right)=0=\lim _{n \rightarrow \infty} \frac{1}{n} H\left(\bigvee_{-m^{\prime} \leq i \leq m^{\prime}} P_{i, 0}\right)
$$

(2)

$$
\begin{aligned}
& H\left(\bigvee_{j w \leq i<(j-1) w+r} P_{i, j} \mid \bigvee_{0 \leq k<j} \bigvee_{k w \leq i \leq 2 m+k w} P_{i, k}\right) \\
& \geq H\left(\underset{j w \leq i<(j-1) w+r}{\bigvee_{i, j}} P_{0 \leq k<j} \bigvee_{k w<i \leq 2 m^{\prime}+k w} P_{i, k}\right),
\end{aligned}
$$

because we condition on more information.

(3) By the same reason, we have

$$
\begin{gathered}
H\left(\underset{(j-1) w-r \leq i \leq j w}{\bigvee} P_{i, j} \mid \bigvee_{0 \leq k<j} \bigvee_{-2 m+k w<i<k w} P_{i, k}\right. \\
\left.\bigvee \underset{-2 m+j w \leq i \leq-2 m+(j-1) w+r}{\bigvee} P_{i, j}\right) \\
\geq H\left(\underset{(j-1) w-r \leq i \leq j w}{\bigvee} P_{i, j} \mid \bigvee_{0 \leq k<j} \bigvee_{-2 m^{\prime}+k w \leq i<k w} P_{i, k}\right. \\
\left.\bigvee_{-2 m^{\prime}+j w \leq i \leq-2 m^{\prime}+(j-1) w+r} P_{i, j}\right) .
\end{gathered}
$$


These observations together with the formula for $H^{m}(\vec{v})$ above shows $H^{m^{\prime}}(\vec{v}) \leq$ $H^{m}(\vec{v})$.

Case 2. $\vec{v}=\eta(1,0)$ for some real $\eta$.

We analogously denote by $H^{m}(\vec{v})$

$$
\lim _{n \rightarrow \infty} \frac{1}{n} H\left(\bigvee_{i=0}^{[n \eta]} \bigvee_{j=-m}^{m} P_{i, j}\right)
$$

We note that

$$
\begin{aligned}
& H^{m^{\prime}}(\vec{v})=\lim _{n \rightarrow \infty} \frac{1}{n} H\left(\bigvee_{i=0}^{[n \eta]} \bigvee_{j=-m}^{m+2\left(m^{\prime}-m\right)} P_{i, j}\right) \\
& =\lim _{n \rightarrow \infty} \frac{1}{n}\left(H\left(\bigvee_{i=0}^{[n \eta]} \bigvee_{j=-m}^{m} P_{i, j}\right)\right. \\
& \left.\quad+H\left(\bigvee_{i=1}^{[n \eta]} \bigvee_{j=m+1}^{m+2\left(m^{\prime}-m\right)} P_{i, j} \mid \bigvee_{i=0}^{[n \eta]} \bigvee_{j=-m}^{m} P_{i, j}\right)\right) \\
& \leq H^{m}(\vec{v})+\lim _{n \rightarrow \infty} \frac{1}{n} \sum_{j=m+1}^{m+2\left(m^{\prime}-m\right)} H\left(\bigvee_{i=0}^{[n \eta]} P_{i, j} \mid \bigvee_{i=0}^{[n \eta]} P_{i, j-1}\right) \\
& \leq H^{m}(\vec{v})+\lim _{n \rightarrow \infty} \frac{1}{n} \sum_{j=m+1}^{m+2\left(m^{\prime}-m\right)} H\left(\bigvee_{i=0}^{r} P_{i, j} \bigvee_{i=[n \eta]-r}^{[n \eta]} P_{i, j}\right) \\
& \leq H^{m}(\vec{v})+\lim _{n \rightarrow \infty} \frac{1}{n} \sum_{j=m+1}^{m+2\left(m^{\prime}-m\right)} 2 r . \tau \\
& =H^{m}(\vec{v})+\lim _{n \rightarrow \infty} \frac{4 r \tau\left(m^{\prime}-m\right)}{n} \\
& =H^{m}(\vec{v}) .
\end{aligned}
$$

Since we have $H^{m^{\prime}}(\vec{v}) \geq H^{m}(\vec{v})$ by definition, the proof is complete.

Corollary 1. If $\vec{v}$ is not a scalar multiple of $(1,0)$, then we have

$$
\begin{aligned}
& \left|\frac{1}{n} H\left(\bigvee_{j=0}^{n-1} \bigvee_{-m+j w \leq i \leq m+j w} P_{i, j}\right)-\frac{1}{n} H\left(\bigvee_{j=0}^{n-1} \bigvee_{-m^{\prime}+j w \leq i \leq m^{\prime}+j w} P_{i, j}\right)\right| \\
& \leq \frac{1}{n} H\left(\bigvee_{m<|i| \leq m^{\prime}} P_{i, 0}\right) \\
& \leq \tau \frac{2\left(m^{\prime}-m\right)}{n} .
\end{aligned}
$$


Theorem 1. $h^{c}(\vec{v})=h(\vec{v})$.

Proof. It is enough to show that $h^{c}(\vec{v})-h(\vec{v})$ is small. If $\vec{v}=(x, y)$ where $y \neq 0$, then by rescaling, we may assume that $\vec{v}=(x, 1)$. Given any $\varepsilon>0$, there exists $\theta$ such that if $\kappa \leq \theta$, then

(i)

$$
\lim _{n \rightarrow \infty} \frac{1}{n} H\left(\bigvee_{0 \leq j<n} \bigvee_{j x_{\kappa} \leq \imath \leq j x_{-\kappa}} P_{\imath, j}\right)<h^{c}(\vec{v})+\varepsilon
$$

(ii) $\left|x_{-\theta}-x_{\theta}\right|<\gamma$ where $\gamma$ satisfies that $\gamma \tau<\varepsilon$. There exists $m_{0}$ such that if $m \geq m_{0}$, then

$$
\lim \frac{1}{n} H\left(\bigvee_{\jmath=0}^{n-1} \bigvee_{-m+\jmath x \leq i \leq m+j x} P_{i, j}\right)=h(\vec{v})
$$

We choose $n_{o}$ such that if $n \geq n_{o}$, then we have

(iii)

$$
h(\vec{v})-\varepsilon<\frac{1}{n} H\left(\bigvee_{0 \leq j \leq n-1} \bigvee_{-m_{\circ}+j x \leq i \leq m_{o}+j x} P_{\imath, j}\right) \leq h(\vec{v})+\varepsilon,
$$

(iv)

$$
h^{c}(\vec{v})-2 \varepsilon<\frac{1}{n} H\left(\bigvee_{o \leq j \leq n-1} \bigvee_{j x_{\theta} \leq i \leq j x_{-\theta}} P_{i, j}\right) \leq h^{c}(\vec{v})+2 \varepsilon
$$

(v)

$$
\begin{gathered}
\frac{1}{n} H\left(\bigvee_{o \leq j<K} \bigvee_{-m_{\circ}+j x<i<m_{\circ}+j x} P_{i, j}\right)<\varepsilon, \text { where } \\
K=\max \left\{j: j\left|x_{\theta}-x\right|<m_{o} \text { and } j\left|x_{-\theta}-x\right|<m_{o}\right\},
\end{gathered}
$$

and

(vi)

$$
\frac{1}{n} H\left(\bigvee_{o \leq j<n} \bigvee_{j x_{\theta} \leq i \leq j x_{-\theta}} P_{i, j}\right) \geq \frac{1}{n} H\left(\bigvee_{0 \leq j<n} \bigvee_{-m_{o}+j x \leq i \leq m_{o}+j x} P_{i, j}\right)
$$


We compute

$$
\begin{aligned}
\left|h^{c}(\vec{v})-h(\vec{v})\right| & \mid \frac{1}{n} H\left(\bigvee_{o \leq j<n} \bigvee_{j x_{\theta} \leq i \leq j x_{-\theta}} P_{i, j}\right) \\
& -\frac{1}{n} H\left(\bigvee_{o \leq j<n} \bigvee_{-m_{o}-j x \leq \imath<m_{o}+j x} P_{i, j}\right) \mid+3 \varepsilon \\
\leq & \mid \frac{1}{n} H\left(\bigvee_{o \leq j<n} \bigvee_{n\left(x_{\theta}-x\right)+j x \leq i \leq n\left(x_{-\theta}-x\right)+j x} P_{i, j}\right) \\
& -\frac{1}{n} H\left(\bigvee_{o \leq j<n} \bigvee_{-m_{o}+j x \leq i<m_{o}+j x} P_{i, j}\right) \mid+3 \varepsilon \\
\leq & \frac{1}{n} H\left(\bigvee_{n\left(x_{\theta}-x\right) \leq i \leq n\left(x_{-\theta}-x\right)} P_{i, o}\right)+3 \varepsilon \\
\leq & \frac{1}{n} \gamma n \tau+3 \varepsilon .
\end{aligned}
$$

Hence we have

$$
\left|h(\vec{v})-h^{c}(\vec{v})\right|<4 \varepsilon .
$$

In the case of $\vec{v}=(x, o)$, it is not hard to see that the idea of the second part of the proof of Lemma 1 combined with the idea of the proof above will give the desired result.

Theorem 2. If $\sum_{m=0}^{\infty} H\left(P_{0,1} \mid \underset{-m \leq i \leq m}{\bigvee} P_{\imath, 0}\right)$ is finite, then we have $h^{c}(\vec{v})=$ $h(\vec{v})$.

Proof. We note that if we choose $M$ so that

$$
\sum_{m=M}^{\infty} H\left(P_{0,1} \mid \bigvee_{-m \leq i \leq m} P_{i, 0}\right)<\varepsilon
$$

then we get

$$
\sum_{k=-m+M}^{m-M} H\left(P_{k, 1} \mid \bigvee_{-m \leq i \leq m} P_{i, 0}\right)<2 \varepsilon
$$


for all $m>M$. Using this, it is easy to see that if $m_{2} \geq m_{1} \geq M$, we have that for any $n$,

$$
\frac{1}{n} H\left(\bigvee_{j=0}^{[n y]} \bigvee_{i=m_{2}+j w}^{-m_{2}+j w} P_{i, j}\right)<\frac{1}{n} H\left(\bigvee_{j=0}^{[n y]} \bigvee_{i=-m_{1}+j w}^{m_{1}+j w} P_{i, j}\right)+2 \varepsilon+\frac{m_{2}-m_{1}}{n} \tau
$$

where $\frac{m_{2}-m_{1}}{n} \tau$ comes from the difference between $\frac{1}{n} H\left(\bigvee_{i=-m_{1}}^{m_{1}} P_{\imath, 0}\right)$ and $\frac{1}{n} H\left(\bigvee_{i=-m_{2}}^{m_{2}} P_{i, 0}\right)$

Hence for a given $\varepsilon>0$, there exist $m_{o}$ as in Theorem 1 such that for a sufficiently large $n$,

$$
\begin{aligned}
\left|h^{c}(\vec{v})-h(\vec{v})\right| & \mid \frac{1}{n} H\left(\bigvee_{o \leq j<n} \bigvee_{n\left(x_{\theta}-x\right)+j \leq \leq i \leq n\left(x_{-\theta}-x\right)+j x} P_{i, j}\right) \\
& -\frac{1}{n} H\left(\bigvee_{o \leq j<n} \bigvee_{-m_{o}+j x \leq i<m_{\circ}+j x} P_{i, j}\right) \mid+3 \varepsilon \\
\leq & \frac{1}{n} \gamma n \tau+2 \varepsilon+3 \varepsilon .
\end{aligned}
$$

Corollary 2. If $V$ is a finitary code with finite expected code length, then $h^{c}(\vec{v})=h(\vec{v})$.

Proof. It is easy to see that a finitary code with finite expected code length satisfies the condition of Theorem 2. See [Pa3].

\section{Main Theorem.}

Let $\lambda=\mu_{1} \times \mu_{2}$. We denote $\sum_{i=0}^{n-1} \varphi_{k}\left(T^{i} z\right)$ by $\varphi_{k}^{n}(z)$ for $k=1$ or 2 and $z \in X_{1}$. Given two partitions, $\beta_{1}$ and $\beta_{2}$, we write $\beta_{1} \leq \beta_{2}$ if $\beta_{2}$ is a finer partition than $\beta_{1}$.

Theorem 3. $h(\widehat{T})=h(T)+h(\vec{v})$ where $\vec{v}=\int \varphi d \mu=\left(\int \varphi_{1} d \mu, \int \varphi_{2} d \mu\right)$.

Proof. Since $\int \varphi d \mu$ is finite, as in the case of a $Z$-valued skewing function, there exists $\varphi^{\prime}$ which is bounded and cohomologous to $\varphi$. Hence we may assume that $\varphi$ is bounded. Let $\left|\varphi_{1}(z)\right| \leq L$ and $\left|\varphi_{2}(z)\right| \leq L$. Suppose $\vec{v}=\int \varphi d \mu=(x, y)$ where $y \neq 0$. We let $\alpha$ denote the generating partition 
of the base. Let $\beta$ denote a partition of $X_{2}$. Both of the partitions $\alpha$ and $\beta$ can be considered in a natural way to be a partition of $X_{1} \times X_{2}$. For a given $z \in X_{1}$, we denote the set $\left\{(z, u): u \in X_{2}\right\}$ by $I_{z}$.

Since

$$
\frac{1}{n} H\left(\bigvee_{i=0}^{n-1} \widehat{T}^{i}(\alpha \bigvee \beta)\right)=\frac{1}{n} H\left(\bigvee_{i=0}^{n-1} \widehat{T}^{i} \alpha\right)+\frac{1}{n} H\left(\bigvee_{i=0}^{n-1} \widehat{T}^{i} \beta \mid \bigvee_{i=0}^{n-1} \widehat{T}^{i} \alpha\right)
$$

and

$$
\frac{1}{n} H\left(\bigvee_{i=0}^{n-1} \widehat{T}^{i} \beta \mid \bigvee_{i=0}^{n-1} \widehat{T}^{i} \alpha\right)=\int \frac{1}{n} H\left(\bigvee_{i=0}^{n-1} \widehat{T}^{i} \beta \mid I_{z}\right) d \mu
$$

we have

$$
\begin{aligned}
& \sup _{\beta} h\left(\widehat{T}, \alpha^{\vee} \beta\right)=\sup _{\beta} \lim _{n \rightarrow \infty} \frac{1}{n} H\left(\bigvee_{i=0}^{n-1} \widehat{T}^{i}\left(\alpha^{\vee} \beta\right)\right) \\
& =h(\widehat{T}, \alpha)+\sup _{\beta_{m}} \lim _{n \rightarrow \infty} \int \frac{1}{n} H\left(\bigvee_{i=0}^{n-1} \widehat{T}^{i} \beta_{m} \mid I_{z}\right) d \mu \\
& =h(\widehat{T}, \alpha)+\lim _{m \rightarrow \infty} \lim _{n \rightarrow \infty} \int \frac{1}{n} H\left(\bigvee_{i=0}^{n-1} \widehat{T}^{i} \beta_{m} \mid I_{z}\right) d \mu,
\end{aligned}
$$

where $\beta_{m}$ denote the partition $\bigvee_{i=-m}^{m} \bigvee_{j=0}^{L-1} P_{i, j}$.

We denote $\lim _{n \rightarrow \infty} \frac{1}{n} H\left(\bigvee_{i=0}^{n-1} \widehat{T}^{i} \beta_{m} \mid I_{z}\right)$ by $h_{z}\left(\widehat{T}, \beta_{m}\right)$.

As in Lemma 1, it is not hard to see that for sufficiently large $m$ and $m^{\prime}$, we have

$$
h_{z}\left(\widehat{T}, \beta_{m}\right)=h_{z}\left(\widehat{T}, \beta_{m^{\prime}}\right) .
$$

We will show that for sufficiently large $m$,

$$
\frac{1}{n} H\left(\bigvee_{i=0}^{n-1} \widehat{T}^{i} \beta_{m} \mid I_{z}\right) \rightarrow h(\vec{v}) \text { as } n \rightarrow \infty \text {, for a.e. } z \in X_{1}
$$

We denote by $x_{\ell}$ the $x$-intercept of a line in $\mathbb{R}^{2}$ passing through $\varphi^{\ell}(z)$ with the same slope as $\vec{v}$. Let

$$
\begin{aligned}
s_{n} & =\max \left\{x_{1}, \ldots, x_{n}\right\} \text { and } \\
t_{n} & =\min \left\{x_{1}, \ldots, x_{n}\right\} .
\end{aligned}
$$

Given $\varepsilon>0$, let $k_{o}$ be the integer such that if $k \geq k_{o}$, then we have 
(i)

$$
\left|h(\vec{v})-\lim _{n \rightarrow \infty} \frac{1}{n} H\left(\bigvee_{0}^{[n y]} \bigvee_{i=-k+j w}^{k+j w} P_{i, j}\right)\right|<\varepsilon .
$$

Given any $\delta>0$ and $\varepsilon>0$, there exists $n_{o}$ such that if $n \geq n_{o}$, then we have

(ii)

$$
\mu E_{1}=\mu\left\{z:\left|\int \varphi d \mu-\frac{1}{n} \varphi^{n}(z)\right|<\delta\right\}>1-\varepsilon
$$

(iii)

$$
\left|h(\vec{v})-\frac{1}{n} H\left(\bigvee_{j=0}^{[n y]} \bigvee_{i=-k_{o}+j w}^{k_{o}+j w} P_{i, j}\right)\right|<\varepsilon
$$

(iv)

$$
\mu E_{2}=\mu\left\{z:\left|h_{z}\left(\widehat{T}, \beta_{k_{o}}\right)-\frac{1}{n} H\left(\bigvee_{i=0}^{n-1} \widehat{T}^{i} \beta_{k_{\circ}} \mid I_{z}\right)\right|<\varepsilon\right\}>1-\varepsilon,
$$

(v) $k_{o}<\frac{\varepsilon}{2} n_{o}$

and

(vi) $\left|s_{n}-t_{n}\right|<2 n \delta$.

We choose $\delta<\varepsilon^{2}$ and choose $n_{o}$ satisfying (ii)-(vi) above. We fix $m_{o}$ such that $k_{o}<(\varepsilon / 2) n_{o}<m_{o}<\varepsilon n_{o}$. For notational convenience, we write $m$ and $n$ instead of $m_{o}$ and $n_{o}$ respectively. We note that

$$
\begin{aligned}
& \bigvee_{j=0}^{n-1} \widehat{T}^{j} \beta_{m} \text { on } I_{Z} \\
& \leq \beta_{m} \bigvee_{F^{\varphi(z)}}\left(\beta_{m}\right) \bigvee F^{\varphi^{2}(z)}\left(\beta_{m}\right) \bigvee \ldots \bigvee_{F^{\varphi^{n-1}}(z)}\left(\beta_{m}\right) \text { on } I_{z} \\
& \leq \bigvee_{j=0}^{\varphi_{2}^{n-1}(z)+L-1} \bigvee_{i=t_{n}-m+j w}^{s_{n}+m+\jmath w} P_{i, j} \text { on } I_{Z} \text {. }
\end{aligned}
$$

Since $t_{n}$ and $s_{n}$ satisfy that

$$
\left|\left(t_{n}+m\right)-\left(s_{n}-m\right)\right|=\left|2 m+t_{n}-s_{n}\right|>|2 m-2 n \delta|>k_{o}
$$

and

$$
\left|\left(s_{n}+m\right)-\left(t_{n}-m\right)\right|<\varepsilon n,
$$


if $z \in E_{1}$, then by our Corollary and (ii), we have

$$
\begin{aligned}
& \left|\frac{1}{n} H\left(\bigvee_{j=0}^{\varphi_{2}^{n-1}(z)+L-1} \bigvee_{i=t_{n}-m+j w}^{s_{n}+m+j w} P_{i, j}\right)-\frac{1}{n} H\left(\bigvee_{j=0}^{[n y]} \bigvee_{i=-k_{o}+j w}^{k_{o}+j w} P_{i, j}\right)\right| \\
& <\frac{1}{n} H\left(\bigvee_{i=t_{n}-m}^{s_{n}+m} P_{i, 0}\right)+\frac{1}{n} H\left(\bigvee_{j=q_{1}}^{q_{2}} \bigvee_{i=t_{n}-m+j w}^{s_{n}+m+j w} P_{i, j}\right) \\
& <\frac{1}{n} \tau \varepsilon n+\frac{1}{n}\left(q_{2}-q_{1}\right) \tau(w+2 r) \\
& <\tau(\varepsilon+\delta(w+2 r)),
\end{aligned}
$$

where $q_{1}=\min \left\{[n y], \varphi_{2}^{n-1}(z)+L-1\right\}$ and $q_{2}=\max \left\{[n y], \varphi_{2}^{n-1}(z)+L-1\right\}$.

Hence we have

$$
\begin{aligned}
& \left|\frac{1}{n} H\left(\bigvee_{i=0}^{n-1} \widehat{T}^{i} \beta_{m} \mid I_{z}\right)-h(\vec{v})\right| \\
& \leq\left|\frac{1}{n} H\left(\bigvee_{i=0}^{n-1} \widehat{T}^{i} \beta_{m} \mid I_{z}\right)-\frac{1}{n} H\left(\bigvee_{j=0}^{[n y]} \bigvee_{i=-k_{o}+j w}^{k_{o}+j w} P_{i, j}\right)\right|+\varepsilon \\
& \leq\left|\frac{1}{n} H\left(\bigvee_{j=0}^{\varphi_{2}^{n-1}(z)+L-1} \bigvee_{i=t_{n}-m+j w}^{s_{n}+m+j w} P_{i, j}\right)-\frac{1}{n} H\left(\bigvee_{j=0}^{[n y]} \bigvee_{i=-k_{o}+j w}^{k_{o}+j w} P_{i, j}\right)\right|+\varepsilon \\
& \leq \tau(\varepsilon+\delta(w+2 r))+\varepsilon .
\end{aligned}
$$

Let $E=E_{1} \cap E_{2}$. If $z \in E$, then by our choice of $m$ and Corollary 1, we have

$$
\begin{aligned}
& \left|\frac{1}{n} H\left(\bigvee_{i=0}^{n-1} \widehat{T}^{i} \beta_{m} \mid I_{z}\right)-h_{z}\left(\widehat{T}, \beta_{m}\right)\right| \\
& \leq\left|\frac{1}{n} H\left(\bigvee_{i=0}^{n-1} \widehat{T}^{i} \beta_{m} \mid I_{z}\right)-\frac{1}{n} H\left(\bigvee_{i=0}^{n-1} \widehat{T}^{i} \beta_{k} \mid I_{z}\right)\right| \\
& \quad+\left|\frac{1}{n} H\left(\bigvee_{i=0}^{n-1} \widehat{T}^{i} \beta_{k} \mid I_{z}\right)-h_{z}\left(\widehat{T}, \beta_{k}\right)\right|+\left|h_{z}\left(\widehat{T}, \beta_{k}\right)-h_{z}\left(\widehat{T}, \beta_{m}\right)\right| \\
& \leq \varepsilon+\varepsilon+\frac{1}{n} m \tau<\varepsilon(2+\tau) .
\end{aligned}
$$

Since $\varphi_{1}$ and $\varphi_{2}$ are bounded, it is easy to see that there exists $\omega$ such that $\left|h_{z}(\widehat{T}, \beta)\right|<\omega$ for all $\beta$ and all $z$. We may also assume that $h(\vec{v})$ is 
bounded above by $\omega$. Now we compute

$$
\begin{aligned}
& \left|\sup _{\beta} \int h_{z}(\widehat{T}, \beta) d \mu-h(\vec{v})\right| \\
& \leq \int_{E}\left|h_{z}\left(\widehat{T}, \beta_{m}\right)-h(\vec{v})\right| d \mu+\sup _{\beta} \int_{E^{c}}\left|h_{z}(\widehat{T}, \beta)-h(\vec{v})\right| d \mu+\varepsilon \\
& \leq \int_{E}\left|h_{z}\left(\widehat{T}, \beta_{m}\right)-\frac{1}{n} H\left(\bigvee_{i=0}^{n-1} \widehat{T}^{i} \beta_{m} \mid I_{z}\right)\right| d \mu \\
& +\int_{E}\left|\frac{1}{n} H\left(\bigvee_{i=0}^{n-1} \widehat{T}^{i} \beta_{m} \mid I_{z}\right)-h(\vec{v})\right| d \mu \\
& +\sup _{\beta} \int_{E^{c}}\left|h_{z}(\widehat{T}, \beta)-h(\vec{v})\right| d \mu+\varepsilon \\
& \leq \varepsilon(2+\tau)+\tau(\varepsilon+\delta(w+2 r))+\varepsilon+4 \omega \varepsilon+\varepsilon \\
& \leq \varepsilon(4+2 \tau+\tau(w+2 r)+4 \omega) \text {. }
\end{aligned}
$$

In the case when $\vec{v}=\int \varphi=\eta(1,0)$ for some real number $\eta$, we need to argue differently. We may assume $\eta>0$. We construct $\varphi^{\prime}$ which is cohomologous to $\varphi$ as follows. Let $\varphi^{\prime}=\left(\varphi_{1}^{\prime}, \varphi_{2}^{\prime}\right)$.

(i) $\varphi_{1}^{\prime}$ takes the values $[\eta]-1,[\eta]$ and $[\eta]+1$

$\varphi_{2}^{\prime}$ takes the values $-1,0,1$.

(ii) In an orbit of a point, $\varphi_{2}^{\prime}$ value, 1 or -1 , follows its value 0 .

(iii) We use the ergodic theorem to construct $\varphi_{2}^{\prime}$ so that it takes the value 0 for all $z^{\prime}$ s except a set of small measure.

Hence we may assume that $\varphi$ satisfies these properties.

We let $\beta_{m}=\bigvee_{i=0}^{[\eta]} \bigvee_{j=-m}^{m} P_{i, j}$. Recall that $r$ denote the size of the block map. As in the previous case,we choose $m_{o}$ so that if $m \geq m_{o}$, then

(i) $m_{o} \geq 10 r$,

(ii) $\left|h(\vec{v})-H^{m}(\vec{v})\right|<\varepsilon$,

(iii) $\mu\left\{z:\left|\sup _{\beta} \int h_{z}(\widehat{T}, \beta)-h_{z}\left(\widehat{T}, \beta_{m}\right)\right|<\varepsilon\right\}>1-\varepsilon$.

We fix $m \geq m_{o}$. We choose $n_{o}$ so that if $n \geq n_{o}$, then

(iv) $\mu\left\{z:\left|\frac{1}{n} H\left(\bigvee_{i=0}^{n-1} \widehat{T}^{i} \beta_{m} \mid I_{z}\right)-h_{z}\left(\widehat{T}, \beta_{m}\right)\right|<\varepsilon\right\}>1-\varepsilon$,

(v) $\mu\left\{z:\left|\frac{1}{n} \sum_{i=0}^{n-1} \varphi\left(T^{i}(z)\right)-\int \varphi d \mu\right|<\varepsilon\right\}>1-\varepsilon$, and

(vi) $\mu\left\{z:\left|\frac{1}{n} \sum_{i=0}^{k} \varphi_{2}\left(T^{i}(z)\right)\right|<\varepsilon\right.$ for all $\left.0 \leq k<n\right\}>1-\varepsilon$.

Let $E$ denote the set satisfying the above conditions, (iii), (iv), (v) and 
(vi). We have $\mu E>1-4 \varepsilon$. Let $z \in E$.

Let

$$
u=\max \left\{\sum_{i=0}^{k} \varphi_{2}\left(T^{i}(z)\right): k=0,1, \ldots, n-1\right\}
$$

and

$$
v=\min \left\{\sum_{i=0}^{k} \varphi_{2}\left(T^{i}(z)\right): k=0,1, \ldots, n-1\right\} .
$$

Since $\eta>0$, there exists $i_{o}=\max \left\{k: \varphi_{1}^{k}(z) \leq i\right\}$ for a.e. $z \in X_{1}$. We denote by $\Psi_{2}^{i}(z)$

$$
\max \left\{\sum_{\zeta=0}^{k} \varphi_{2}\left(T^{\zeta}(z)\right): 0 \leq k \leq i_{o}, i-[\eta] \leq \varphi_{1}^{k}(z) \leq i\right\} .
$$

Now we compute

$$
\begin{aligned}
& \frac{1}{n} H\left(\bigvee_{j=-m}^{m} \bigvee_{i=0}^{\varphi_{1}^{n}(z)} P_{i, j}\right) \\
& =\frac{1}{n} H\left(\bigvee_{j=-m+u}^{m+u} \bigvee_{i=0}^{\varphi_{1}^{n}(z)} P_{i, j}\right) \\
& \leq \frac{1}{n} H\left(\bigvee_{i=0}^{\varphi_{1}^{n}(z)} \bigvee_{j=-m+\Psi_{2}^{i}(z)}^{u+m} P_{i, j}\right) \\
& \leq \frac{1}{n}\left(H\left(\bigvee_{i=0}^{\varphi_{1}^{n}(z)} \bigvee_{j=-m+\Psi_{2}^{i}(z)}^{m+(z)} P_{i, j}\right)+2 \cdot 2(\varepsilon n) \cdot \tau \cdot r\right) \\
& \leq \frac{1}{n} H\left(\bigvee_{i=0}^{n-1} \widehat{T}^{i} \beta_{m} \mid I_{z}\right)+4 \varepsilon r \tau .
\end{aligned}
$$

The second to the last inequality is clear because by the condition (i) on $m_{o}$, we have

$$
\begin{aligned}
& H\left(\bigvee_{i=0}^{\varphi_{1}^{n}(z)} \bigvee_{j=-m+\psi_{2}^{i}(z)}^{u+m} P_{i, j} \bigvee_{i=0}^{\varphi_{1}^{n}(z)} \bigvee_{j=-m+\psi_{2}^{i}(z)}^{m+\psi_{2}^{i}(z)} P_{i, j}\right) \\
& \leq H\left(\bigvee_{j=m}^{u+m} \bigvee_{i=0}^{r-1} P_{i, j} \bigvee \bigvee_{j=v+m}^{u+m} \bigvee_{i=\varphi_{1}^{n}(z)-r+1}^{\varphi_{1}^{n}(z)} P_{i, j}\right)
\end{aligned}
$$




$$
\leq u \cdot r \cdot \tau+(u-v) \cdot r \cdot \tau
$$

Since the following inequality is also true

$$
\begin{aligned}
& \frac{1}{n} H\left(\bigvee_{i=0}^{n-1} \widehat{T}^{i} \beta_{m} \mid I_{z}\right) \\
& \leq \frac{1}{n} H\left(\bigvee_{j=-m+v}^{m+u} \bigvee_{i=0}^{\varphi_{1}^{n}(z)} P_{i, j}\right) \\
& =\frac{1}{n} H\left(\bigvee_{j=-m}^{m+u-v} \bigvee_{i=0}^{\varphi_{1}^{n}(z)} P_{i, j}\right) \\
& \leq \frac{1}{n} H\left(\bigvee_{j=-m}^{m} \bigvee_{i=0}^{\varphi_{1}^{n}(z)} P_{i, j}\right)+\frac{2}{n}(u-v) r \cdot \tau \\
& =\frac{1}{n} H\left(\bigvee_{\jmath=-m+u}^{m+u} \bigvee_{i=0}^{\varphi_{1}^{n}(z)} P_{i, j}\right)+4 \varepsilon r \tau,
\end{aligned}
$$

we have

$$
\left|\frac{1}{n} H\left(\bigvee_{i=0}^{n-1} \widehat{T}^{i} \beta_{m} \mid I_{z}\right)-\frac{1}{n} H\left(\bigvee_{-m+u}^{m+u} \bigvee_{\imath=0}^{\varphi_{1}^{n}(z)} P_{i, j}\right)\right|<4 \varepsilon r \tau .
$$

We note that

$$
\frac{1}{n} H\left(\bigvee_{-m+u}^{m+u} \bigvee_{i=0}^{\varphi_{1}^{n}(z)} P_{i, j}\right)=\frac{\varphi_{1}^{n}(z)}{n} \frac{1}{\varphi_{1}^{n}(z)} H\left(\bigvee_{-m+u}^{m+u} \bigvee_{i=0}^{\varphi_{1}^{n}(z)} P_{i, j}\right)
$$

converges to $h(\vec{v})$.

As in the case of $\vec{v}=\int \varphi d \mu=(x, y)$ where $y \neq 0$, it is now clear that

$$
\left|\sup _{\beta} \int h_{z}(\widehat{T}, \beta) d \mu-h(\vec{v})\right|
$$

can be made arbitraily small.

Similarly we can prove the following theorem.

Theorem 4. If $\sum_{m=0}^{\infty} H\left(P_{0,1} \mid \underset{-m \leq i \leq m}{\bigvee} P_{\imath, 0}\right)$ is finite, then we have $h(\widehat{T})=$ $h(T)+h(\vec{v})$ where $\vec{v}=\int \varphi d \mu=\left(\int \varphi_{1} d \mu, \int \varphi_{2} d \mu\right)$.

The following Corollaries are also almost immediate from the proof of Theorem 3. 
Corollary 3. If $\sum_{m=0}^{\infty} H\left(P_{0,1} \mid \bigvee_{-k \leq \jmath \leq k} \underset{-m \leq \imath \leq m}{\bigvee} P_{i, j}\right)$ is finite for some $k$, then we have $h(\widehat{T})=h(T)+h(\vec{v})$ where $\vec{v}$ is given as above.

Corollary 4. If a fiber $Z^{2}$-action, $F$, satisfies the condition of Corollary 3 after a linear transformation by a matrix $A$ in $S L(2, Z)$, that is, $A \circ F$ satisfies the condition, then we have the above formula in Corollary 3 for the entropy.

\section{References}

Ab, Ro] L.M. Abramov and V.A. Rohlin, The entropy of a skew product of measure preserving transformations, AMS Translations, Ser. 2.

[Ad] R. Adler, A note on the entropy of skew product transformations, Am. Math. Soc., 4 (1963), 665-669.

[He] G.A. Hedlund, Endomorphrsms and automorphisms of the shift dynamıcal system, Math. Syst. Theor., 3 (1969), 320-375.

[Li] D. Lind, personal communication.

$\mathrm{Ma}, \mathrm{Ne}]$ S.B. Marcus and S. Newhouse, Measure of maximal entropy for a class of skew products, Springer Lect. Notes Math., 729 (1979), 105-125.

[Mi1] J. Milnor, On the entropy geometry of cellular automata, Complex Systems, 2 (1988), 357-386.

[Mi2] Directional entropies of cellular automation-maps, Nato ASI Series, vol. F20, (1986), 113-115.

[Pa1] K.K. Park, On the continuity of directional entropy, Osaka J. Math., 31 (1994), 613-628.

[Pa2] - A counter example of the entropy of the skew product, preprint.

[Pa3] Continuity of directional entropy for a class of $Z^{2}$-actions, J. Korean Math. Soc., 32 (1995), 573-582.

[Si] Y. Sinai, An answer to a question by J. Mılnor, Comment. Math. Helv., 60 (1985), 173-178.

[Th] J.P. Thouvenot, personal communication.

Received February 10, 1993 and revised June 24, 1993. This research has been supported in part by NSF DMS 8902080 and GARC-KOSEF.

AJOU UNIVERSITY

SuwON, 441-749

KoreA 




\section{PACIFIC JOURNAL OF MATHEMATICS}

Volume $172 \quad$ No. $1 \quad$ January 1996

A class of incomplete non-positively curved manifolds

BRIAN BOWDITCH

The quasi-linearity problem for $C^{*}$-algebras

41

L. J. BUNCE and JOHN DAVID MAITLAND WRIGHT

Distortion of boundary sets under inner functions. II

Jose Luis Fernandez Perez, Domingo Pestana and José Rodríguez

Irreducible non-dense $A_{1}^{(1)}$-modules

VJACHESLAV M. FUTORNY

$M$-hyperbolic real subsets of complex spaces

101

Giuliana Gigante, Giuseppe Tomassini and Sergio Venturini

Values of Bernoulli polynomials

ANDREW GRANVILLE and ZHI-WEI SUN

The uniqueness of compact cores for 3-manifolds

LUKE HARRIS and PETER SCOTT

Estimation of the number of periodic orbits

BOJU JIANG

Factorization of $p$-completely bounded multilinear maps

Christian Le MERdy

Finitely generated cohomology Hopf algebras and torsion

JAMEs Peicheng LiN

The positive-dimensional fibres of the Prym map

JUAN-CARLOS NARANJO

Entropy of a skew product with a $Z^{2}$-action

KYEWON KOH PARK

Commuting co-commuting squares and finite-dimensional Kac algebras

TAKASHI SANO

Second order ordinary differential equations with fully nonlinear two-point boundary 255 conditions. I

H. BEVAN THOMPSON

Second order ordinary differential equations with fully nonlinear two-point boundary conditions. II

H. BEVAN THOMPSON

The flat part of non-flat orbifolds

FENG XU 